\section{MODEL IDEAS: FroM STEM \\ Cell Simulation to FLOATING ART WORK}

Jane Prophet, Computing Department, Goldsmiths College, University of London, SE14 6NW, U.K. E-mail: $<$ jane@janeprophet.com>.

See $<$ www.mitpressjournals.org/toc/leon/44/3 $>$ for supplemental files associated with this issue.

Submitted: 30 April 2010

Abstract

This paper discusses the role of models in the development of an interactive artwork made as the result of interdisciplinary collaboration. A variety of different types of model were used, each with different functions and status to the team. Keywords: architecture, cellular automata, collaboration, interarchitecture, cellular automata, collaboration, inter-
active, interdisciplinary, LEDs, model, modelscope, simulation, stem cell

CELL [1] was an interdisciplinary collaboration that ran from 2003-2008 and explored the ways that research into adult stem cells re-addresses the complexity of human biology. Medical scientist Neil Theise, a researcher in adult stem cells, worked with artist Jane Prophet, mathematician Mark d'Inverno, computer scientist Rob Saunders and curator Peter Ride. One aim was to visualize new and contentious theories of stem cell behavior and to feed these visualizations back into scientific research Another was to generate a range of art works, under-pinned by an understanding of cellular activity.

Throughout our collaboration, we wondered not only at the science of stem cells but also at the role that models and visualizations have played in the transformation of scientific beliefs. This contribution will introduce and contextualize the role of the model in the design of the art installation Net Work, currently in development, which is based on our mathematical model of how stem cells function in the adult human body [2].

\section{Description of Net Work}

Net Work will be a large, highly interactive art installation constructed from 100 buoys fitted with RGB LED marine lanterns, placed at 1-meter intervals to form a $10 \times 10$ grid. The lanterns are connected to a computer running a cellular automata (CA) program. The lanterns' behavior (the color they display, which can change in real time) is based on a CA derived from our stem cell model. Each buoy is a node on the grid, undulating on moving water, acting like a cell. The LEDs display changing colors that correspond to the behavioral state of the buoys/cells. Each cell's state depends on both its environment (the movement of the water, detected by tilt sensors and light levels, affected by users shining torches on the grid at night) and the state of neighboring cells. By constantly checking for inputs and changing the colors they display as a result, the grid acts as a living network of cells. The rules that the buoys contain encode biological predictions about how stem cells behave.

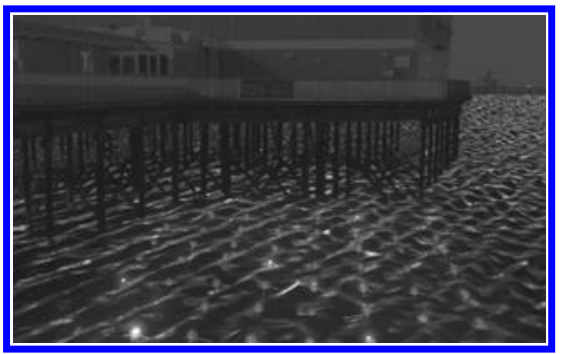

Fig. 1. Digital sketch of Net Work (C) Jane Prophet. Animation by Gideon Corby.)

Net Work integrates two types of audience interaction, the first when the audience is physically proximate, the second, for a remote audience affecting the work over the Internet. We hope that Net Work will stimulate interest in two strands of contemporary scientific research - the definition and development of stem cells in relation to computational models of complex systems - and the process of scientific discovery itself.

In the background, but of profound importance to us as an interdisciplinary team, is the role that our formal language models, computer science (CS) and artistic visualizations have played in the transformation of our scientific beliefs, and this is the focus of the remainder of this paper. The 'public engagement with science' project that runs alongside the installation demonstrates the role that some easily understood models have had in fostering scientific orthodoxy, and online tools will enable people to build computational models to form and test hypotheses. Our hope is that participants will explore the attributes of compelling and useful models and begin to understand that scientific progress is a creative act, involving the building and interpreting of tractable models that act as metaphors for the intractable world. For us, an essential part of CS is this process of model development and implementation.

\section{The Importance of 'Scaling'}

Our core hypothesis is that models do not represent certainties (or truths) and our question is largely of how that impacts on our understandings of science.
Throughout our collaboration we have relied heavily on different types of model. Our plain English models (linguistic models) use words to describe how stem cells behave in the adult human body. Our linguistic model was developed from research data based on observations from across the stem cell research community about stem cell behavior but gave priority to Theise's theories. From this d'Inverno wrote a formalist mathematical model, which we used as the basis for a computer simulation. This displayed the behaviors we had defined using images that looked like cells (a representational model, but one that represented function as much as form). These were equally 'models of a theory'-Theise's theory of stem cells [3].

Art school training informed the particular set of assumptions about models that I brought with me to the project. Scale models were familiar to me, and their 'partial' or approximate value always acknowledged (material scale models may be an accurate, down-sized representation of a target system or sculpture, but are rarely faithful in every aspect - for example the volume and shape of a sculpture may be faithfully down-sized but the material qualities are often very different, with say, bronze, represented by clay). In my art practice a model is often based on a sketch. It is a three-dimensional object, either real or virtual that is usually 'smaller than' the 'final art piece' and it is made of less expensive, more throw-away, materials.

Fig. 2. Initial sketch of Net Work (C) Jane Prophet)

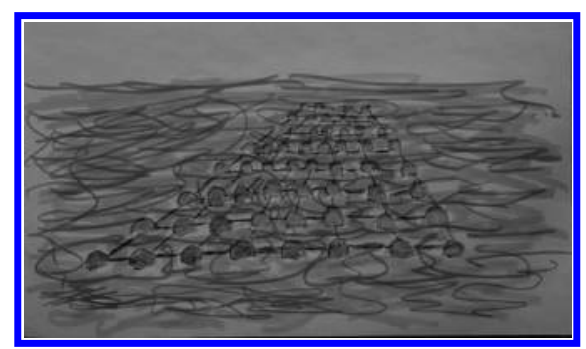

Scale models and more idealized (sketchier) material models are used to explore and imagine a range of possible final outcomes for an artwork. The reduced size of the model is more malleable and frees me both physically and financially, enabling me to try out numerous configurations and materials. In my mind's eye I move between the model and the final version at a much larger size. I also shift scales within the modelmaking process itself. Frequently I make a close-up or 'zoomed in' physical mod- 
el, producing certain components at a much bigger size, to test my production technique at the 'final' scale and/or with the final materials. These models are more like prototypes. We 'scaled' in this way when we made a nine buoy prototype for Net Work to explore the way the lanterns displayed the CA.

As we worked, we moved between CA models displayed on computer screens, to the linguistic model, to the prototype, to the data visualizations. These different models had a function similar to that described by architectural theorist Albena Yaneva. In her writing about the use of models by architects, she says that the common architectural practice of 'scaling' (literally moving back and forth between large and small scale models) "can be considered as an experimental situation in the sense that it is subjected to constant and wellequipped observation of possible consequences of acting on scale models ... [when architects 'scale' they do so as] an exploratory move by probing in trial and error fashion, or as a systematic test aiming for an intended outcome to be confirmed or disconfirmed" [4]. Architects use a modelscope to look inside models to see things that are not visible directly from outside the foam model.

When first working in the CELL team, I was struck by the centrality of the microscope in Theise's research; this instrument was essential in order to view cell structures that are invisible to the human eye. The microscopic study of tissue, on glass slides, brings with it problems associated with viewing 'dead' tissue: important interactions between cells, and between cells and the dynamic and complex physical and chemical environment of the surrounding body, characteristic of living tissues, are unobservable. The tissue on the slide is frozen in time and place-immobile, boundaried, isolated, dead. We were sure that new hypotheses about stem cell behavior would be prompted if we could only look at living stem cells in their 'natural' environment. As this is not yet possible we were prompted to produce simulations of cell systems.

Our simulation enabled us to prioritize the relationship of the part to the whole, the single cell to the organism. The real-time three-dimensional simulation built by Saunders allowed us to 'scale' like the architects, to use zoom controls on the graphic user interface to look at the system close up and from farther away, and to make graphic repre-

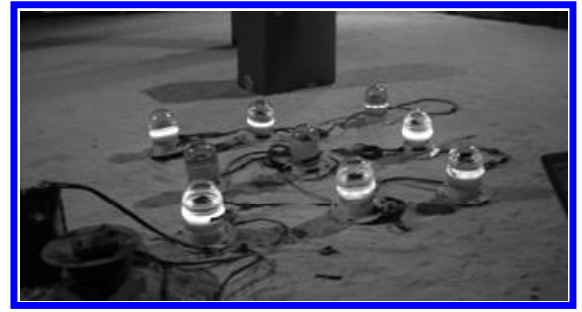

Fig. 3. Testing the RGB LED marine lanterns as part of a full size model or prototype. (C) Jane Prophet. Produced by the Net Work team.)

sentations of cells reveal their underlying data when clicked. Our intention was to use this 'scaling' action to observe patterns of cell behavior. As Theise has stated, our interpretations and understanding of complex organisms are impacted by the scale at which we can observe; "[a]t the gross level, bodies are things. At the microscopic level, they are not things, but systems of interacting cells. Likewise, cells appear as things on the microscopic scale, but disappear on the way to the nanoscale as their biomolecular subunits become the target of observation" [5]. So, for us to develop new hypotheses about stem cell behavior, the ability to 'scale' is essential; "[f]or the observer of a complex system, the nature of the system depends on the scale of observation (i.e., how close up one gets to it). What appears to be a unified, functional entity on the macroscale is also an extraordinarily dynamic, organizational dance of myriad, separate individuals on the microscale"[6].

Yaneva also advocates the model as a vehicle that can incorporate features that can then be observed (under a variety of conditions) in order to test hypotheses. For her, a key function of models is their use as "important tools for shared cognition: architects think of the building by modeling." Yaneva stresses that this 'thinking by modeling' is "not a free intuitive creation"- the model, from the very start, is a product of constraints.

The development of a plain English (or linguistic) model of stem cell behavior was fraught with difficulty, not least around our use of language [7]. However, our text-based model became (as Yaneva might say of the architect's foam model) "an object of collective experience, which is visible for many actors at the same time". Yaneva's subjects viewed their physical model using a modelscope and then made changes on a bigger physical model for others to see; we used the linguistic model in a similar way. We shared it electronically, each adding comments to reveal our different interpretations of the model. Using this low-tech text-based approach, we shared what we 'saw' with each of our collaborative partners, and as a result the cognitive properties of the team were changed

The visualization of our mathematical model had a similar function; its purpose was to (re)present the formal model using images (a 2D real-time graphic display; a 3D version with photorealistic cells; a CA version). As experts from different disciplines observed these visual models and discussed their observations, once again the cognitive properties of the team changed. To take our virtual model and make it physical by producing Net Work we have another opportunity to develop and expand our cognitive model. Net Work is a model, but it is not simply a massively scaled-up representation of a microscopic complex system of cells. Nor is it a linguistic representation of those cells. Nor is it a data model. It is more a model that has the capacity to condense ideas [8], an Aristotelian idealization "that strips away, in our imagination, all properties from a concrete object that we believe are not relevant to the problem at hand. This allows us to focus on a limited set of properties in isolation" [9] — in this case stem cell behavior.

\section{References and Notes}

* This paper was presented as a contributed talk at Arts | Humanities | Complex Networks a Leonardo satellite symposium at NetSci2010. See <http://artshumanities.netsci2010.net>

1. J. Prophet. Accessed 26 April 2010, $<$ http://www.janeprophet.com/cell.html $>$.

2. Mark d'Inverno, Neil Theise and Jane Prophet, "Mathematical modelling of stem cells: a complexity primer for the stem cell biologist" in C. Potten, J. Watson, R. Clarke, and A. Renehan eds. Tissue Stem Cells: Biology and Applications (Oxord: Oxford University Press, 2006).

3. For a nuanced discussion of models in science visit the Stanford Online Encyclopedia of Philosophy. Accessed 26 April 2010, $<$ http://plato.stanford.edu/entries/modelsscience/\#RepModIModPhe>.

4. Albena Yaneva, "Scaling Up and Down: Extraction Trials in Architectural Design," in Social Studies of Science.

5. Mark d'Inverno and Jane Prophet, "Creative conflict in interdisciplinary collaboration interpretation, scale and emergence" in E. Edmonds and R. Gibson eds., Interaction: Systems, Theory and Gibson eds., Interaction: Systems,
Practice (New York: ACM, 2005).

6. Neil D. Theise "Cell Doctrine in a Complex and Uncertain World: Time for Reappraisal?" Cloning and Stem Cells 7, No. 4, 2005 pp. 209-214.

7. Mark d'Inverno and Jane Prophet [5]

8. Jane Prophet and Nina Wakeford, "A conversation about models and prototypes" in Hazel Gardiner and Charlie Gere, eds., Art Practice in a Digital Culture (Surrey, UK: Ashgate).

9. Stanford Online Encyclopedia of Philosophy [3] 